\title{
Optimizing Nondecomposable Data Dependent Regularizers via Lagrangian Reparameterization Offers Significant Performance and Efficiency Gains
}

\author{
Sathya N. Ravi, Abhay Venkatesh, Glenn M. Fung, Vikas Singh \\ sathya@uic.edu, abhayvenkate@wisc.edu, gfung@amfam.com, vsingh@ biostat.wisc.edu
}

\begin{abstract}
Data dependent regularization is known to benefit a wide variety of problems in machine learning. Often, these regularizers cannot be easily decomposed into a sum over a finite number of terms, e.g., a sum over individual example-wise terms. The $F_{\beta}$ measure, Area under the ROC curve (AUCROC) and Precision at a fixed recall (P@R) are some prominent examples that are used in many applications. We find that for most medium to large sized datasets, scalability issues severely limit our ability in leveraging the benefits of such regularizers. Importantly, the key technical impediment despite some recent progress is that such objectives remain difficult to optimize via backpropapagation procedures. While an efficient general-purpose strategy for this problem still remains elusive, in this paper, we show that for many data-dependent nondecomposable regularizers that are relevant in applications, sizable gains in efficiency are possible with minimal code-level changes; in other words, no specialized tools or numerical schemes are needed. Our procedure involves a reparameterization followed by a partial dualization - this leads to a formulation that has provably cheap projection operators. We present a detailed analysis of runtime and convergence properties of our algorithm. On the experimental side, we show that a direct use of our scheme significantly improves the state of the art IOU measures reported for MSCOCO Stuff segmentation dataset.
\end{abstract}

\section{Introduction}

Data dependent regularization is a mature and effective strategy for many problems in machine learning. In semisupervised learning (Corduneanu and Jaakkola 2006), the marginal distribution of the examples may serve to influence the estimation of the conditional distribution and in SVMs, one could optimize the maximum relative margin based on the data distribution, rather than the $a b$ solute margin. In modern deep learning, data-dependent regularization is routinely used in both explicit and implict ways. A regularizer can take the form of certain geometric or clustering-type constraints (Lezama et al. 2018; Zhu et al. 2018) on the representations that are learned by the network - using the distribution overlap between different classes (Rippel et al. 2015) or seeking decorrelated autoencoder latent codes (Cheung et al. 2014). On the other hand, artificial data corruption is also a form of regularization dropout induces a regularization on the Fisher information matrix of the samples (Wager, Wang, and Liang 2013). More recently, the results in (Mou et al. 2018) offer a nice treatment of the form of data-dependent regularization that emerges from popular methods such as batch norm and AdaGrad.

From Decomposable to Nondecomposable datadependent regularizers. A number of data-dependent regularizers described above can be written as a sum of individual example-wise estimates of the regularizer. This setting is desirable because in order to run a SGD type algorithm, we simply pick a random sample to get an unbiased estimate of the gradient. But a number of application domains often necessitate a regularization criteria that may not decompose in this manner. In such settings, a random sample (minibatch) of the dataset does not provide us an unbiased gradient - biased gradients are known to adversely impact both the qualitative and quantitative performance of the training procedure, see (Chen and Luss ).

Why are Nondecomposable regularizers relevant? Consider the situation where we would like to ensure that the performance of a statistical model is uniformly good over groups induced via certain protected attributes (such as race or gender), see Figure 1. Or alternatively, we want that when updating an algorithm in a manufacturing process, the new system's behavior should mostly remain similar with respect to some global measures such as makespan (Limentani et al. 2005). (Cotter et al. 2019) shows applications of various shape constraints that are associated with set functions. And finally, when pooling datasets from multiple sites, global characteristics of Precision-Recall should be (approximately) preserved across sites (Zhou et al. 2017). Essentially, these applications suggest that the performane of a model in expectation (on the entire population), does not automatically guarantee that the model will perform well on specific subgroups. Motivated by these issues encountered in various real world problems, recently (Cotter et al. 2018) presents a comprehensive study of the computational aspects of learning problems with rate constraints - there, the constrained setting is preferred (over penalized setting) due to 

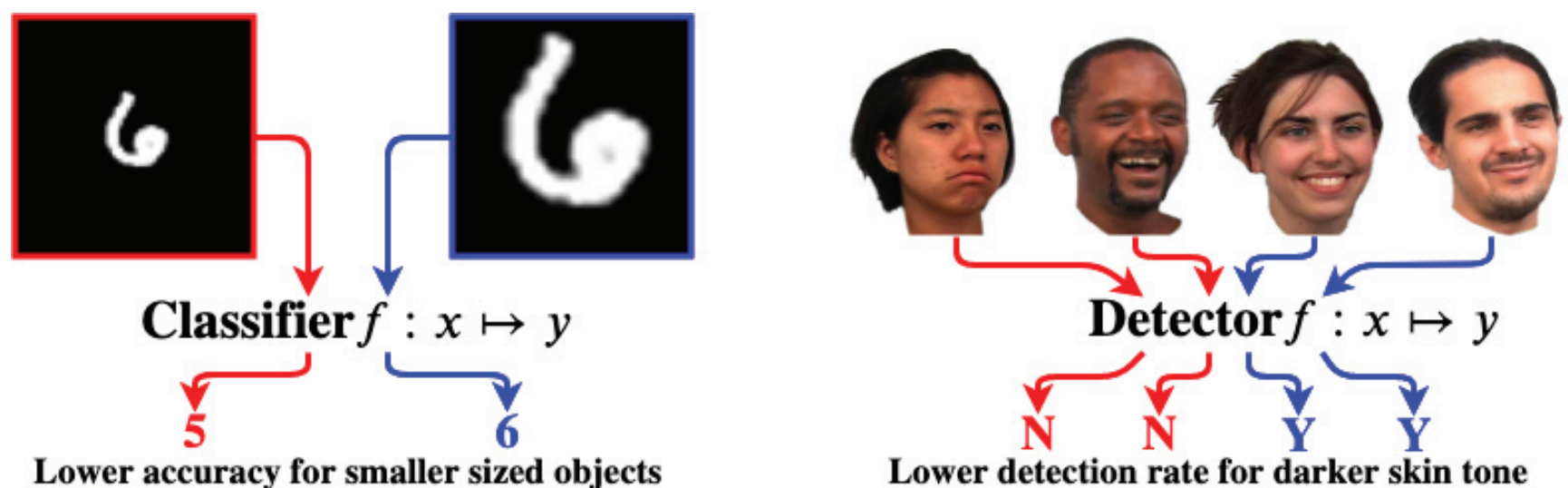

Figure 1: We illustrate the need for data dependent regularization. The performance of an image classifier (left) and face detector (right) can vary significantly depending on the size (small objects get misclassified) and skin tone (weak or no detection power for darker skin) of the data. A data dependent term can explicitly make the classifier/detector to behave similarly for various subgroups in the population.

several reasons including its ease of use for a practitioner. The authors show that for a general class of constraints, a proxy-Lagrangian based method must be used because the Lagrangian is not optimal. This raises the question whether there exist a broad class of data-dependent nondecomposable functions for which the regularized/penalized formulation based on standard Lagrangian schemes may, in fact, be effective and sufficient. In this paper, we will address this question with simple examples shortly.

Examples in statistics. Nondecomposable regularization, in the most general sense, has also been studied from the statistical perspective, and is often referred to as shape constraints (Groeneboom and Jongbloed 2014). The need for shape constraints arise in clinical trials and cohort studies of various forms in the competing risk model, formalized using local smooth function theory, see Chapter 5 in (Daimon, Hirakawa, and Matsui 2018) and (Chenxi and Fine 2012). While several authors have studied specific forms of this problem, the literature addressing the computational aspects of estimation schemes involving such regularizers is sparse, and even for simple objectives such as a sparse additive model, we find that results have appeared only recently (Yin and Yu 2018). Leveraging these ideas to train richer models of the forms that we often use in modern machine learning, establishing their convergence properties, and demonstrating their utility in real world applications is still an open problem.

Our Contributions. We first reparameterize a broad class of nondecomposable data-dependent regularizers into a form that can be efficiently optimized using first order methods. Interestingly, this reparameterization naturally leads to a Lagrangian based procedure where existing SGD based methods can be employed with little to no change, see Figure 2. While recent results suggest that optimizing nondecomposable datadependent regularizers may be challenging (Cotter et al. 2018), our development shows that a sizable subclass of such regularizers indeed admit simple solution schemes. Our overall procedure comes with convergence rate guarantees and optimal per-iteration complexity. On the MSCOCO stuff segmentation dataset, we show that a direct use of this technique yields significant improvements to the state of the art, yielding a mean IoU of 0.32. Pytorch code for our experiments, the extended version of the paper containing proofs and results can be found in https://github.com/abhay-venkatesh/f1-cv.

\section{Preliminaries}

Basic notations. We assume that the training data is given as pairs of $(x, y) \sim \mathcal{D}$ where the joint distribution $\mathcal{D}$ is unknown. Here, $x, y$ are random variables that represent examples (e.g., images) and labels respectively. We make no assumptions on $x$ and $y$ in that the marginal distributions of $x$ and $y$ can be discrete or continuous. Our goal is to estimate a function $f: x \mapsto y$ that achieves the minimum error measured using a specified loss function on the empirical/observed samples. We will use $W=\left\{w_{1}, w_{2}, \ldots, w_{l+1}\right\}$ to represent the trainable parameters of a feedforward neural network with $l+1$ layers, with nonlinear activation functions. The output $W(x)$ of the function computed by the neural network $W$ may be used to construct the classification or regression function $f$.

Nondecomposability. Now, suppose there exists a function $\varphi$ such that $\varphi \circ \mathcal{D}=: s$ is a random variable called "shape" in a similar sense as described above (Groeneboom and Jongbloed 2014). Our notation is suggestive of the fact that $s$ is nondecomposable, i.e., the value $s_{i}$ for individual datapoint/example $i$ may depend on the entire (empirical) distribution of $\mathcal{D}$. Moreover, this implies that without any further assumptions, any loss function $\mathcal{R}(\cdot)$ on $W(x)$ used to learn a model $g$ to predict $s$ cannot be represented as a decomposable function with respect to the training data $x, y$, see (Narasimhan, Vaish, and Agarwal 2014; Sanyal et al. 2018).

As briefly noted in Section 1, nondecomposability poses unique challenges in designing algorithms for learning models with a large number of parameters. In most cases, exist- 


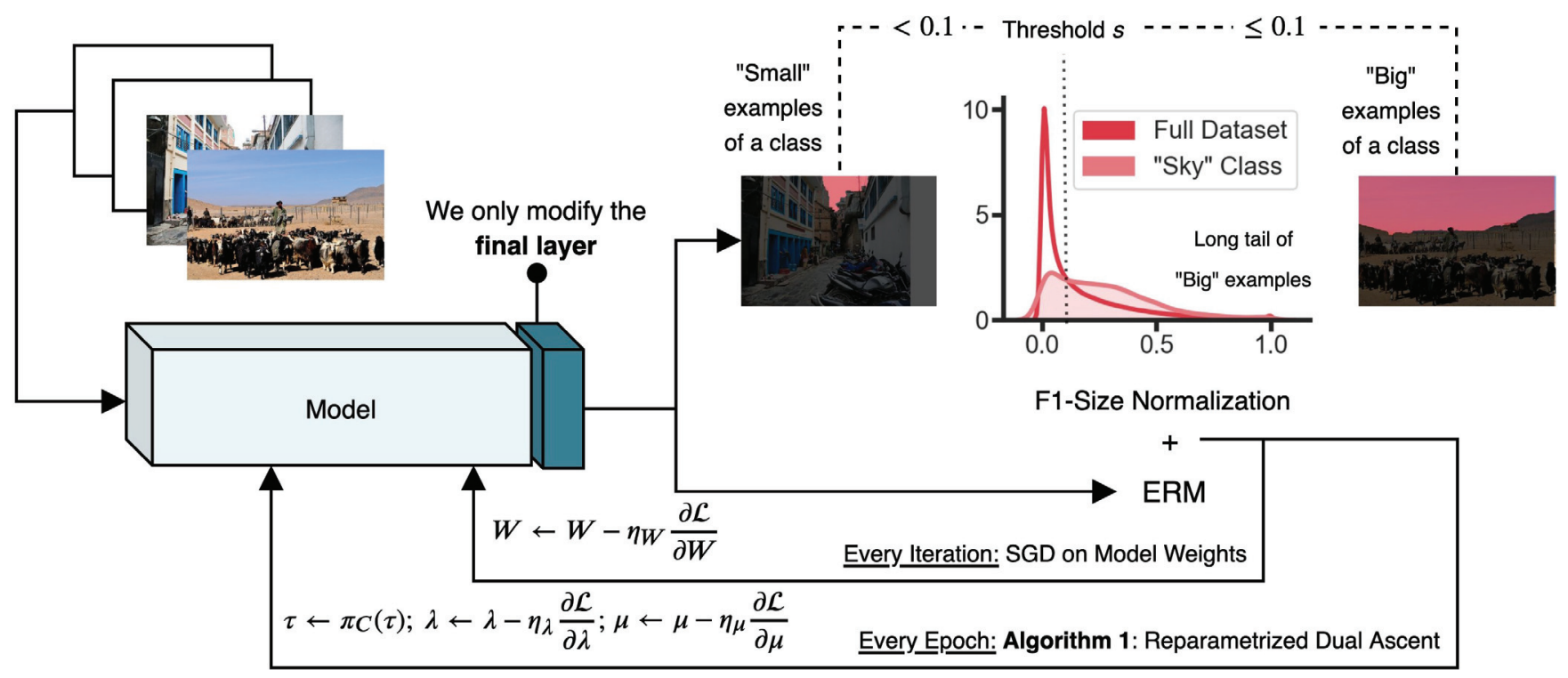

Figure 2: Our proposed training pipeline for optimizing nondecomposable $\mathcal{S}$-Measures.

ing backpropagation based techniques may not be directly applicable and require careful modifications (Zhang et al. 2018). In other cases, they may require significant computational overhead due to expensive projection operations (Kar, Narasimhan, and Jain 2014) or likelihood computations (Ye et al. 2012). Before we present our model, we now briefly describe two fundamental tasks that are, by and large, the most frequently used modules in vision based pipelines to see the benefits of nondecomposability.

Example 1. Object Detection seeks to simultaneously localize and classify objects in an image using a bounding box for each object (Zhao et al. 2019). Naturally, since the bounding boxes are rectangular, the data-dependent regularizer $s$ is fully determined by the size of the objects. In other words, $s$ here becomes the area of the bounding box of the object. Here, we may use the knowledge of $s$ to learn $f$ that can perform equally well over all sizes present in the training data. But even though we may simply use class activation maps to compute the empirical distribution of $s$ (Zhou et al. 2016), the loss functions $\mathcal{R}(s)$ that are commonly used are nondecomposable as we will see shortly.

Example 2. Semantic Segmentation seeks to assign each pixel of an image to a class (Chen et al. 2018). Recent works suggest that in order to train a model $f$, we may choose a model whose complexity strongly depends on the number of pixels in the images, see (Liu, Deng, and Yang ) for a recent survey. Unfortunately, these methods use up/downsampling and so the learned representations do not directly offer immunity to variations in how much of the image is occupied by each class (i.e., size).

Remark 1. We use these two examples to illustrate the applicability of our developments - since the use case corresponds to size, we may assume that the "shape" random variable is discrete. To that end, we will use $\mathcal{S}$ to denote the countable scoring set or the support of $s$ with $|\mathcal{S}|<\infty$.

\subsection{Incorporating $\mathcal{S}$-measures for Optimization}

Let us write down a formulation which incorporates our shape regularizer. The objective function is a sum of two terms: (1) a decomposable Empirical Risk Minimization (ERM) term to learn the optimal function which maps $x$ to $y$ and (2) a nondecomposable, data dependent $\mathcal{S}$-measure regularizer term for $s$ from $x$. In particular, for a fixed $\alpha>0$, we seek to solve the following optimization problem,

$$
\min _{W} \overbrace{\frac{1}{N} \sum_{i=1}^{N} \operatorname{loss}\left(W ; x_{i}, y_{i}\right)}^{\text {ERM }}+\alpha \overbrace{\mathcal{R}(W ; \varphi \circ \hat{D})}^{\mathcal{S} \text {-Measure }},
$$

where $\hat{D}$ represents the empirical distribution, $x_{i} \in \mathbb{R}^{d}$ and $y_{i} \in \mathbb{R}^{k}$ with $i=1, \ldots, N$ denoting training data examples. We let loss $(\cdot)$ to be any standard loss function such as a cross entropy loss, hinge loss and so on. To keep the presentation and notations simple but nonetheless illustrate our main algorithmic developments, we will assume that the $\mathcal{S}$-Measure is given by $F_{\beta}$ metric (Lipton, Elkan, and Naryanaswamy ) while noting that the results apply directly to other measures such as R@P, P@R and can be easily generalized to other nondecomposable metrics such as AUCROC, AUCPR, using the Nyström method with no additional computational overhead (Eban et al. 2016).

Remark 2. For simplicity, we will suppress the dependence of $f$ computed by the parameters $W$ in our objective function (1). For example, if $f$ is represented using a deep neural network with weights $W$, then both terms in (1) may be nonconvex. 


\section{Reparameterizing $\mathcal{S}$-Measures}

Since any backpropagation based procedure can easily handle decomposable terms, let us ignore the ERM term in our objective function (1) for the moment, and focus on the second term. Specifically, in this section, we will focus on the $F_{\beta}$ metric as a placeholder for $\mathcal{S}$-measures. and show that there is a reformulation which will enable a backpropagation based procedure to solve our problem (1). For simplicity, let us assume that $s \in\{+1,-1\}$ and $\beta=1$. The appendix shows how to extend our approach to any finite $|\mathcal{S}|$ and $\beta \in(0,1]$ noting that in various applications in Section $4,|\mathcal{S}|=2$ suffices and is already quite effective (e.g., the learned model works whether $s_{i}$ is +1 or -1 ).

The starting point of our technical development is the following result,

Observation 1 (Restated from (Eban et al. 2016)). If $f$ is linear, i.e., $y=W \cdot x$, we can represent the $\mathcal{S}$-measure term as a Linear Program (LP) as shown in equation (13) in (Eban et al. 2016).

Moving forward from Observation 1. In principle, one could use standard LP solvers to solve the LP in (Eban et al. 2016). But this requires instantiating the constraint matrix (linear in the number of samples). This is impractical - the alternative in (Eban et al. 2016) is to use an iterative ascent method. In general this will also require a projection scheme, e.g., solving a QP. While more attractive than using an offthe-shelf method, both options may need cubic time.

$$
\begin{aligned}
& \text { Problem (2): Slightly adapted form of LP in (Eban et al. 2016) } \\
& \min _{\phi, \tau, w, \epsilon} n \epsilon+\sum_{i \in S^{+}} \tau_{i}+\sum_{i \in S^{-}} \phi_{i} \\
& \text { s.t. } \tau_{i} \leq \epsilon, \tau_{i} \leq w_{l+1} \cdot a_{l}\left(x_{i}\right) ; \forall i \in S^{+} \\
& \phi_{i} \geq 0, \phi_{i} \geq \epsilon+w_{l+1} \cdot a_{l}\left(x_{i}\right) ; \forall i \in S^{-} \\
& \sum_{i \in S^{+}} \tau_{i}=1 \quad \epsilon \geq 0 \text {. }
\end{aligned}
$$

Further, this discussion of the computational issues only pertains to a linear objective - the setting expectedly becomes much more challenging for non-convex objectives common in deep learning architectures.

\subsection{Simplify and Reparameterize}

We will first generalize the construction in (Eban et al. 2016) to be more amenable to non-linear functions that one often estimates with DNN architectures. Note that since our data-dependent $\mathcal{S}$-measures are specified on the input distribution of examples or representations derived from the transformations induced by the network $W$, we may denote representations in general as $a_{l}\left(x_{i}\right)$ : in other words, the $l-$ th layer provides us a representation of example $i$. We define $S^{+}:=\left\{i: s_{i}=+1\right\}$ (and similarly $S^{-}$) with $\left|S^{+}\right|=n$, and $n+\left|S^{-}\right|=N$, calculated using only the training data samples $X:=\left\{x_{i}\right\}, Y:=\left\{y_{i}\right\}, i=1, \ldots, N$. We will still focus on the nondecomposable term but bring in the DNN loss when we describe the full algorithm.
Problem 3: a simplified LP

$$
\begin{array}{cl}
\min _{\tau, w, \epsilon} & n \epsilon+\sum_{i \in S^{-}} \max \left(0, \epsilon+w_{l+1} \cdot a_{l}\left(x_{i}\right)\right) \\
\text { s.t. } & \tau_{i} \leq \epsilon, \tau_{i} \leq w_{l+1} \cdot a_{l}\left(x_{i}\right) ; \forall i \in S^{+} \\
& \sum_{i \in S^{+}} \tau_{i}=1 \quad \epsilon \geq 0 .
\end{array}
$$

Eliminating redundancy in Problem (2). First, notice that we can eliminate the $\sum_{i \in S^{+}} \tau_{i}$ term from the objective function in (2a) since any feasible solution has to satisfy the first constraint in (2d). Second, using the definition of hinge loss, we can eliminate the $\phi_{i}$ terms in the objective by replacing $\phi_{i}$ with $\max \left(0, \epsilon+w_{l+1} \cdot a_{l}\left(x_{i}\right)\right)$. This rearrangement leads to a LP in Problem (3).

Remark 3. The choice of $\phi$ to be the hinge loss is natural in the formulation of $F_{\beta}$ metric. Our Algorithm 1 can easily handle other commonly used loss functions such as Square, Cross Entropy, and Margin.

Reparameterization via Partial Dualization. Problem (3) has a objective which is expressed as a sum over finite number of terms. But overall, the problem remains difficult because the constraints (3b) are nonlinear and problematic. It turns out that a partial dualization - for only the problematic constraints - leads to a model with desirable properties. Let us dualize only the $\tau_{i} \leq w_{l+1} \cdot a_{l}\left(x_{i}\right)$ constraints in (3b) using $\lambda_{i}$, and the equality constraint in (3c) using $\mu$, where $\lambda_{i}$ and $\mu$ are the dual variables. This yields the Lagrangian,

$$
\begin{aligned}
& L:=n \epsilon+\sum_{i \in S^{-}} \max \left(0, \epsilon+w_{l+1} \cdot a_{l}\left(x_{i}\right)\right) \\
& +\mu\left(\sum_{i \in S^{+}} \tau_{i}-1\right)+\sum_{i \in S^{+}} \lambda_{i}\left(\tau_{i}-w_{l+1} \cdot a_{l}\left(x_{i}\right)\right) .
\end{aligned}
$$

We can denote the set $C:=\left\{\left(\tau_{1}, \tau_{2}, \ldots, \tau_{n}, \epsilon\right): \tau_{i} \leq \epsilon, \epsilon \geq\right.$ $0, i=1, \ldots, n\}$. With this notation, our final optimization problem for the $\mathcal{S}$-measure can be equivalently written as,

$$
\max _{\lambda \geq 0, \mu} \min _{(\tau, \epsilon) \in C, W} L(\tau, w, \epsilon, \lambda, \mu) .
$$

We will shortly describe some nice properties of this model.

Relation to "Disintegration". While procedurally the derivation above is based on numerical optimization, the form in (5) is related to disintegration. Disintegration is a measure theoretic tool that allows us to rigorously define conditional probabilities (Faden and others 1985). As an application, using this technique, we can represent any (also, nondecomposable) measure defined over the graph of a convex function using (conditional) measures over its faces (Caravenna and Daneri 2010). Intuitively, at the optimal solution of (5), the dual multipliers $\lambda_{i}$ can be thought of a representation of the "disintegrated" $\mathcal{S}$-measure, as seen in the last term in (4). In words, if we can successfully disintegrate the $\mathcal{S}$-measure, then there exists a loss function that can be written in the weighted ERM form such that the optimal solutions of the resulting ERM and that of the nondecomposable loss coincide. 


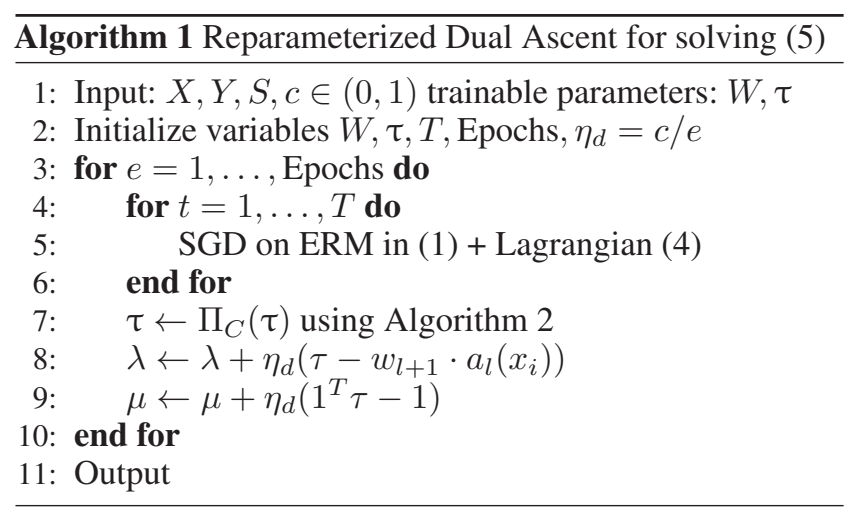

But in general, Lagrangians are difficult to use for disintegration because $\lambda_{i}^{\prime}$ s need not sum to one; and more importantly, when the optimal solution is in the interior of the feasible set in Prob. (3), then $\lambda_{i}=0 \forall i$ by complementarity slackness. This means that the decomposition provided by the $\lambda_{i}^{\prime}$ s need not be a nontrivial (probability) measure in a rigorous sense.

\subsection{Core benefit of Reparameterization: Efficient Projections will become possible}

The previous section omitted description of any real benefits of our reparameterization. We now describe the key computational benefits.

Can we perform fast Projections? Yes, via Adaptive Thresholding. Observe that for the formulation in (5) to offer any meaningful advantage, we should be able to show that the Lagrangian can indeed be efficiently minimized with respect to $\tau, \epsilon, W$. This will crucially depend on whether we can project on to the set $C$ defined above. We now discuss that this holds for different batch sizes.

Fast projections when batch size, $B$ is 2 . Let us denote by $B$, the minibatch size used to solve (5) and consider the setting where we have $S^{+}$and $S^{-}$as used in Probs. (2)-(3). In this case, we can randomly draw a sample $i$ from $S^{+}$and another $i^{\prime}$ from $S^{-}$. Observe that only one coordinate of $\tau$, namely $\tau_{i}$, changes after the primal update. Hence, the projection $\Pi_{C}$ is given by the following simple rule: after the primal update, if $\epsilon<0$, we simply set $\epsilon=0$ leaving $\tau_{i}$ unchanged; on the other hand if $\epsilon>0$, then we have two cases: Case (1) If $\tau_{i} \leq \epsilon$, then do nothing, and Case (2) If $\tau_{i} \geq \epsilon$, then set $\tau_{i}=\epsilon=\left(\tau_{i}+\epsilon\right) / 2$. In other words, the projection is extremely easy.

Remark 4. We can indeed use multiple samples (and take the average) to compute gradients of weights $W, \epsilon$, but only update one $\tau_{i}$ with this scheme.

Generalizing the projection algorithm to larger batch sizes: $B>2$. Note that projections onto sets defined by linear inequality constraints can be performed using standard (convex) quadratic programming solvers (Amos and Kolter 2017). But in the worst case, such solvers use Primal-Dual methods that have a time complexity of $O\left(n^{3}\right)$. It is not

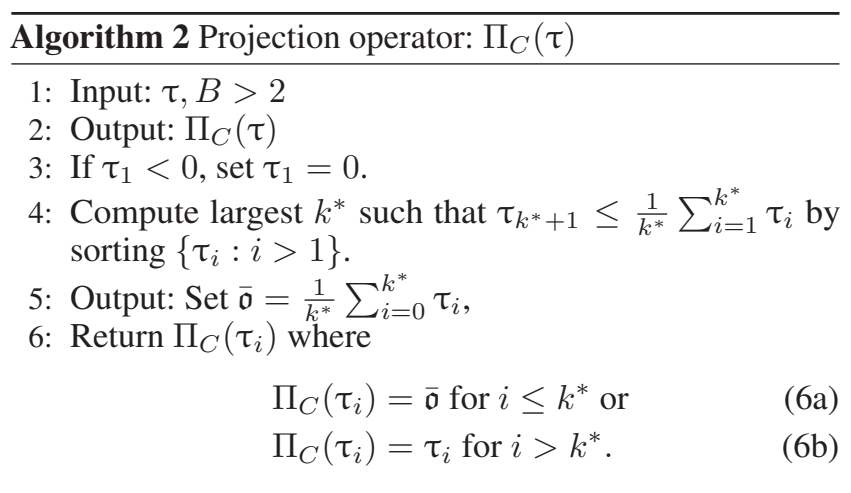

practical to use such solvers efficiently in end-to-end training of widely used neural network architectures. It turns out that a much simpler scheme will work well. Define projection variables $\tau:=[\epsilon ; \tau] \in C$, and $\gamma:=[\delta ; \gamma] \in \mathbb{R}^{n+1}$. The Euclidean projection amounts to solving the following QP:

$$
\Pi_{C}(\gamma)=\arg \min _{\tau}\|\tau-\gamma\|_{2}^{2} \quad \text { s.t. } \quad \tau \in C .
$$

Our projection algorithm to solve (7) is given in Algorithm 2 which requires only a sorting oracle. The following lemma shows the correctness of Algorithm 2.

Lemma 1. There exists an $O(B \log B)$ algorithm to solve (7) that requires only sorting and thresholding operations.

\subsection{Obtaining a numerical optimization scheme}

With the results in the preceding section, we now describe below an efficient approach to solve (1).

Reparameterized Dual Ascent. Using the projection operator above (Alg. 2), our final algorithm to solve $\mathcal{S}$-Measures regularized problem (1) is given in Algorithm 1 where we use $\eta_{d}$ to denote the dual step size. The following theorem characterizes the convergence rate of Algorithm 1.

Theorem 2. Assume that $\left\|\nabla_{W} \operatorname{loss}\left(W ; x_{i}, y_{i}\right)\right\|_{2} \leq G_{1}$, and $\operatorname{Var}_{i}\left(\left\|\nabla_{W} \operatorname{loss}\left(W ; x_{i}, y_{i}\right)\right\|_{2}\right) \leq \sigma$ in the ERM term in Prob. (1). Then, Alg. 1 converges to a $\epsilon$-approximate (local) solution of Prob. (1) in $O(1 / \sqrt{T})$ iterations.

Remark 5. (Implementation details of Alg. 2.) In the appendix, based on Lemma 1, we describe a one pass scheme to compute $k^{*}$ in Step 4 of Alg. 2. So, the time complexity of Alg. 2 is $O(B \log B)$.

Computational Complexity. Assume that each gradient computation is $O(1), \mathrm{Alg}$. 1 requires $T=O\left(1 / \epsilon^{2}\right)$ iterations to compute a local $\epsilon$-optimal solution (inner loop). We use a constant number of epochs $E$ (outer loop), so the total cost is $O\left(E\left(1 / \epsilon^{2}+n \log n\right)\right)$. Please see appendix for more details.

Discussion. The reader will notice that the projection step in Algorithm 1 is outside the inner loop, whereas classical Lagrangian based methods guarantee convergence when the projection is performed in the inner loop. One advantage of having the projection outside the inner for loop is that 

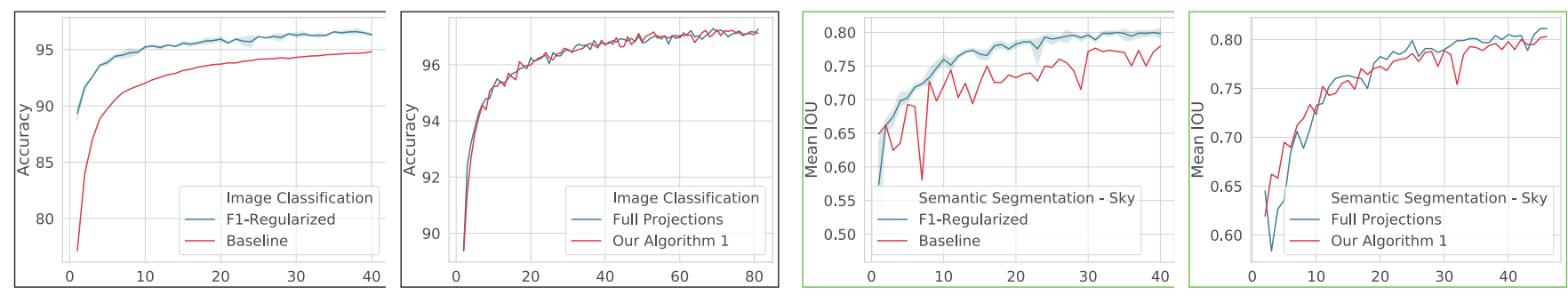

Figure 3: (From Left) Performance of our Reparameterization on $\mathcal{S}$-MNIST (Cols. 1\&2) and MS-COCO class "Sky" (Cols. 3\&4) datasets: $\mathbf{x}$-axis denotes the Epoch in all the plots. Observe that across both the datasets, Reparameterized Dual Ascent Algorithm (red) 1: (i) (Cols. 1\&3) obtains high quality solutions than the Baseline (blue) (Long, Shelhamer, and Darrell 2015); (Cols. 2\&4) and requires significantly less projections (once every epoch from Theorem 2).

SGD type methods allow for faster optimization of the Lagrangian with respect to the primal variables $W$. That is, it is now known that constant step size policies guarantee faster convergence of the inner for loop under some structural assumptions (Dieuleveut, Durmus, and Bach 2017). In any case, we give a detailed description of our Algorithm 1 in the appendix with a discussion of the trade-off between using projections in the inner for loop versus the outer for loop in Algorithm 1.

\subsection{Approximating $\Pi_{C}$ for Backpropagation}

Recall that our primary goal is to solve our problem (5) using backpropagation based methods. In the previous sections, we argued that SGD can be used to solve (5). In particular, we showed in Theorem 2 that Algorithm 1 requires fewer projections compared to the classical PGD algorithm. It turns out that the simplicity of the projection operator $\Pi_{C}$ can be exploited to avoid the double for loops in Algorithm 1, thus enabling backpropagation based training algorithms to be effectively deployed. Details are in the appendix.

\section{Experimental Evaluations}

Overview. We show experiments on three different tasks: $\mathcal{S}$-Measures defined using the $F_{1}$ metric can be efficiently optimized using our Reparameterized Dual Ascent Algorithm 1 in large scale vision problems while strongly improving the empirical performance. The first set of experiments is designed to show that the $F_{1}$ metric based objectives may benefit existing classification models using convex classifiers. The second set of experiments further evaluates the performance of Alg. 1 for nonconvex models. Our goal here is to show that nondecomposable regularizers can be used to stabilize models without sacrificing the overall accuracy. In the third set of experiments, we show that architectures used for Semantic Segmentation can be trained using Alg. 1. In all our experiments, we used ADAM optimizer (with default parameters) to train with primal step sizes of $\eta_{\tau}=\eta_{\epsilon}=\eta_{W}=10^{-2}$, and dual step sizes of $\eta_{\lambda}=10^{-3}$, and $\eta_{\mu}=10^{-5}$. We report the results for the regularization parameter taking values $\alpha=\left\{10^{-2}, 10^{-3}, 10^{-4}\right\}$. Baseline corresponds to $\alpha=0$.

Experiment 1) Improving Linear Classifiers using $\mathcal{S}$-Measures Dataset. Consider a dataset with distinct classes: MNIST with 10 classes (digits). In MNIST, the size of the foreground (pixels with nonzero intensities) is about the same for all images (and each class) in MNIST - the distribution of foreground size $s^{\prime}$ is unimodal (see appendix). To see the benefits of $\mathcal{S}$-measures, we create an augmented dataset called $\mathcal{S}$-MNIST. We added a set of images in which the foreground is double the original size in MNIST simply by rescaling the foreground to get a two mode $s^{\prime}$.

How to obtain size $\mathbf{s}$ ? To get $s \in\{0,1\}$, we can simply threshold $s^{\prime}$ using the empirical mean.

Model and Training. We used a single layer neural network $f$ to predict $y$ from $x$ but added an extra node to account for the $\mathcal{S}$-Measure term in (1). That is, our total loss is the sum of the softmax cross-entropy ERM loss and the $\mathcal{S}$-Measure (using $F_{1}$ metric). We trained using Alg. 1 for 40 epochs with a minibatch size of 100 .

Results. We see the benefits provided by the $F_{1}$ metric as a data-dependent regularizer in Fig. 3. Column 1 shows that our model is uniformly more accurate than the baseline throughout the training process. Moreover, Column 2 compares Alg. 1 with classical dual ascent procedures from (Cotter et al. 2018). Here, full projections refers to computing $\Pi_{C}(\cdot)$ after every inner iteration in Alg. 1. Clearly, we can see that Algorithm 1 obtains high quality solutions but needs one projection operation every epoch.

Takeaway \#1. Models obtained using Alg. 1 are more accurate and stable for linear classifiers.

Experiment 2) Improving one class Segmentation using $\mathcal{S}$-Measures. We consider the task of the pixel-wise contextual labeling of an image. We found that the "sky" category in the MSCOCO stuff (2017) dataset (Caesar, Uijlings, and Ferrari 2018) has a high variance over the samples in terms of size: so, taking this property into account seems sensible. So, we use only the images in the "sky" category (Caesar, Uijlings, and Ferrari 2018).

How to obtain size $s$ ? We first computed the empirical distribution of number of pixels $s^{\prime}$ that are labeled as sky in the training set. We then pick the mean of $s^{\prime}$ to be the threshold to obtain a binary $s$.

Model and Training. We utilize SegNet (Badrinarayanan, Kendall, and Cipolla 2017; Shah 2017), a deep encoderdecoder architecture for semantic segmentation for its simple design and ease of use. As before, we add a fully-connected 

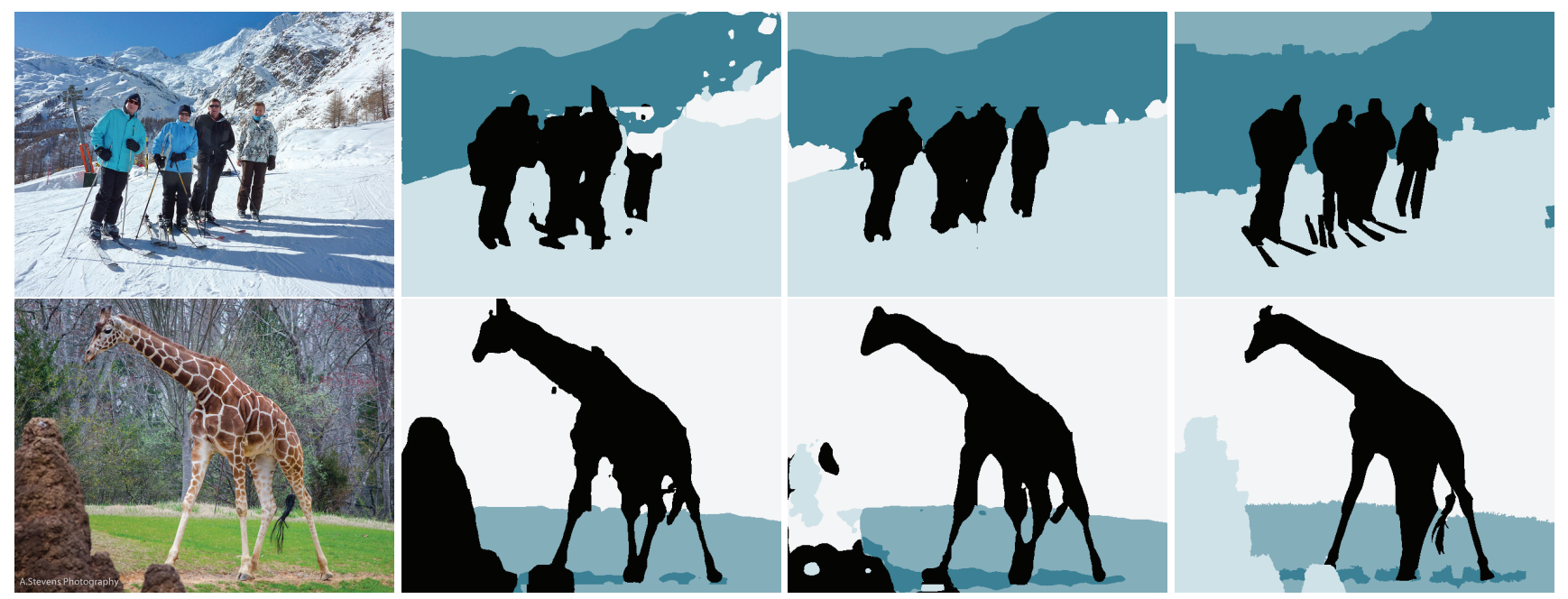

Figure 4: Qualitative results on MSCOCO stuff segmentation benchmark. Colors (except black) indicate different "stuff" categories in an image. From left to right: original image, baseline, our result and ground truth.

layer at the end of the model to incorporate the $\mathcal{S}$-Measure specified by $F_{1}$ metric. We trained our models for 40 epochs with a learning rate of 0.01 and batch size 7 .

Results. Figure 3 Column 3 shows our results averaged over the thresholds $\{0.55,0.6,0.65\}$. We can see that our models uniformly outperforms the baseline with a $80 \%$ mean IOU (over $77.6 \%$ baseline). Furthermore, observe that our Alg. 1 is much more stable than the baseline throughout the training process even in nonconvex settings while requiring the fewest projection steps.

Takeaway \#2. Performance boosts from the F1-regularizer carries over from the simple convex classification task to the pixel-wise classification task with a deep neural network.

Experiment 3) Improving Semantic Segmentation with Nondecomposable Regularizers Dataset. We are now ready to consider semantic segmentation with multiple classes in each image. Our dataset consists of $164 \mathrm{~K}$ images that belong to any of the "stuff" classes in the MSCOCO "Stuff" dataset (Caesar, Uijlings, and Ferrari 2018). We downsampled the training images to $106 \times 160$ size to reduce training time.

How to obtain size $s$ ? The volume of stuff $c$ denoted by $s^{\prime c}$ is measured by the number of pixels that belong to $c$. We observed that $s^{\prime c}$ is close to $0 \%$ for most $c$ (see appendix). So, we picked a threshold of $\approx 0.05$ to obtain a binary $s^{c} \in\{0,1\}$ for each class $c$. Then, we use the majority vote provided by all classes present in an image to obtain $s \in\{0,1\}$ for individual images - corresponding to "big/small". That is, if the majority of the classes present inside the image are "big" (as determined by the threshold $s=0.05$ ), then we assigned the image to be "big" and vice-versa.

Model and Training. We use DeepLabV3+ (Chen et al. 2018) for training. DeepLabV3+ is a popular model for semantic segmentation (needs no CRF post-processing) and can be trained end-to-end. We used a minibatch size of 144 for baseline and 120 for our $F_{1}$ regularized models.
Results. Figure 4 Column 1 shows the quantitative results of our experiment averaged over the thresholds $\{0.01,0.03,0.05,0.07,0.09\}$. Columns 2-4 shows some of our qualitative results. See appendix for quantitative results on MSCOCO stuff segmentation benchmark. Our mean IOU improves upon the state of the art reported in (Chen et al. 2018) by $\approx 10 \%$ on MSCOCO $164 \mathrm{~K}$ while being stable.

Takeaway \#3. On the MS COCO 164K Stuff dataset, we achieve state of the art results with 0.32 Mean IOU (vs. 0.30 current state of the art mean IOU in (Chen et al. 2018)).

\section{Conclusions}

While nondecomposable data-dependent regularizers are variously beneficial and needed in a number of applications, their benefits cannot often be leveraged in large scale settings due to computational challenges. Further, the literature provides little guidance on mechanisms to utilize such regularizers within the deep neural network architectures that are commonly used in the community. In this paper, we showed how various nondecomposable regularizers may indeed permit highly efficient optimization schemes that can also directly benefit from the optimization routines implemented in mature software libraries used in vision and machine learning. We provide a technical analysis of the algorithm and show that the procedure yields state of the art performance for a semantic segmentation task, with only minimal changes in the optimization routine.

\section{Acknowledgments}

Research supported by American Family Insurance, UW CPCP AI117924, NSF CCF \#1918211, NIH R01 AG062336 and R01 AG059312, NSF CAREER award RI\#1252725, and start-up funds from the University of Illinois at Chicago. We thank Shailesh Acharya and Songwong Tasneeyapant for discussions and help with some of the experiments. 


\section{References}

Amos, B., and Kolter, J. Z. 2017. Optnet: Differentiable optimization as a layer in neural networks. In Proceedings of the 34th ICML-Volume 70. JMLR. org.

Badrinarayanan, V.; Kendall, A.; and Cipolla, R. 2017. Segnet: A deep convolutional encoder-decoder architecture for image segmentation. IEEE transactions on pattern analysis and machine intelligence 39(12):2481-2495.

Caesar, H.; Uijlings, J.; and Ferrari, V. 2018. Coco-stuff: Thing and stuff classes in context. In CVPR.

Caravenna, L., and Daneri, S. 2010. The disintegration of the lebesgue measure on the faces of a convex function. Journal of Functional Analysis.

Chen, J., and Luss, R. Stochastic gradient descent with biased but consistent gradient estimators. arXiv:1807.11880.

Chen, L.-C.; Zhu, Y.; Papandreou, G.; Schroff, F.; and Adam, H. 2018. Encoder-decoder with atrous separable convolution for semantic image segmentation. In $E C C V$.

Chenxi, L., and Fine, J. 2012. Smoothed nonparametric estimation for current status competing risks data. Biometrika.

Cheung, B.; Livezey, J. A.; Bansal, A. K.; and Olshausen, B. A. 2014. Discovering hidden factors of variation in deep networks. arXiv preprint arXiv:1412.6583.

Corduneanu, A., and Jaakkola, T. 2006. Data dependent regularization. Semi-Supervised Learning 163-182.

Cotter, A.; Jiang, H.; Wang, S.; Narayan, T.; Gupta, M.; You, S.; and Sridharan, K. 2018. Optimization with nondifferentiable constraints with applications to fairness, recall, churn, and other goals. arXiv preprint arXiv:1809.04198.

Cotter, A.; Gupta, M.; Jiang, H.; Louidor, E.; Muller, J.; Narayan, T.; Wang, S.; and Zhu, T. 2019. Shape constraints for set functions. In ICML.

Daimon, T.; Hirakawa, A.; and Matsui, S. 2018. An Introduction to Dose-Finding Methods in Early Phase Clinical Trials. Springer.

Dieuleveut, A.; Durmus, A.; and Bach, F. 2017. Bridging the gap between constant step size stochastic gradient descent and markov chains. arXiv preprint arXiv:1707.06386.

Eban, E. E.; Schain, M.; Mackey, A.; Gordon, A.; Saurous, R. A.; and Elidan, G. 2016. Scalable learning of nondecomposable objectives. arXiv preprint arXiv:1608.04802.

Faden, A. M., et al. 1985. The existence of regular conditional probabilities: necessary and sufficient conditions. The Annals of Probability.

Groeneboom, P., and Jongbloed, G. 2014. Nonparametric Estimation under Shape Constraints: Estimators, Algorithms and Asymptotics. Cambridge Series in Statistical and Probabilistic Mathematics.

Kar, P.; Narasimhan, H.; and Jain, P. 2014. Online and stochastic gradient methods for non-decomposable loss functions. In Advances in NeurIPS.

Lezama, J.; Qiu, Q.; Musé, P.; and Sapiro, G. 2018. Olé: Orthogonal low-rank embedding-a plug and play geometric loss for deep learning. In Proceedings of the IEEE Conference on CVPR.
Limentani, G. B.; Ringo, M. C.; Ye, F.; Bergquist, M. L.; and McSorley, E. O. 2005. Beyond the t-test: statistical equivalence testing.

Lipton, Z. C.; Elkan, C.; and Naryanaswamy, B. Optimal thresholding of classifiers to maximize 11 measure. In Joint European Conference on Machine Learning and Knowledge Discovery in Databases. Springer.

Liu, X.; Deng, Z.; and Yang, Y. Recent progress in semantic image segmentation. Artificial Intelligence Review.

Long, J.; Shelhamer, E.; and Darrell, T. 2015. Fully convolutional networks for semantic segmentation. In IEEE conference on CVPR.

Mou, W.; Zhou, Y.; Gao, J.; and Wang, L. 2018. Dropout training, data-dependent regularization, and generalization bounds. In ICML.

Narasimhan, H.; Vaish, R.; and Agarwal, S. 2014. On the statistical consistency of plug-in classifiers for nondecomposable performance measures. In Advances in NeurIPS.

Rippel, O.; Paluri, M.; Dollar, P.; and Bourdev, L. 2015. Metric learning with adaptive density discrimination. arXiv preprint arXiv:1511.05939.

Sanyal, A.; Kumar, P.; Kar, P.; Chawla, S.; and Sebastiani, F. 2018. Optimizing non-decomposable measures with deep networks. Machine Learning.

Shah, M. P. 2017. Semantic segmentation architectures implemented in pytorch. https://github.com/meetshah1995/pytorchsemseg.

Wager, S.; Wang, S.; and Liang, P. S. 2013. Dropout training as adaptive regularization. In Advances in NeurIPS.

Ye, N.; Chai, K. M. A.; Lee, W. S.; and Chieu, H. L. 2012. Optimizing f-measures: a tale of two approaches. In Proceedings of the 29th ICML.

Yin, J., and Yu, Y. 2018. Convex-constrained sparse additive modeling and its extensions. CoRR.

Zhang, X.; Liu, M.; Zhou, X.; and Yang, T. 2018. Faster online learning of optimal threshold for consistent f-measure optimization. In Advances in NeurIPS.

Zhao, Z.-Q.; Zheng, P.; Xu, S.-t.; and Wu, X. 2019. Object detection with deep learning: A review. IEEE transactions on neural networks and learning systems.

Zhou, B.; Khosla, A.; Lapedriza, A.; Oliva, A.; and Torralba, A. 2016. Learning deep features for discriminative localization. In Proceedings of the IEEE conference on CVPR.

Zhou, H. H.; Zhang, Y.; Ithapu, V. K.; Johnson, S. C.; Singh, V.; et al. 2017. When can multi-site datasets be pooled for regression? hypothesis tests, $\ell_{2}$-consistency and neuroscience applications. In ICML.

Zhu, W.; Qiu, Q.; Huang, J.; Calderbank, R.; Sapiro, G.; and Daubechies, I. 2018. Ldmnet: Low dimensional manifold regularized neural networks. In The IEEE Conference on CVPR. 